\title{
Automatic Mitral Annulus Tracking In Volumetric Ultrasound Using Non-rigid Image Registration
}

\author{
Henri De Veene ${ }^{1}$, Philippe B. Bertrand ${ }^{2}$, Natasa Popovic ${ }^{3}$, Pieter M. Vandervoort ${ }^{2}$, \\ Piet Claus ${ }^{3}$, Matthieu De Beule ${ }^{1}$ and Brecht Heyde ${ }^{3}$
}

\begin{abstract}
Analysis of mitral annular dynamics plays an important role in the diagnosis and selection of optimal valve repair strategies, but remains cumbersome and time-consuming if performed manually. In this paper we propose non-rigid image registration to automatically track the annulus in $3 D$ ultrasound images for both normal and pathological valves, and compare the performance against manual tracing. Relevant clinical properties such as annular area, circumference and excursion could be extracted reliably by the tracking algorithm. The root-mean-square error, calculated as the difference between the manually traced landmarks (18 in total) and the automatic tracking, was $1.96 \pm 0.46 \mathrm{~mm}$ over 10 valves (5 healthy and 5 diseased) which is within the clinically acceptable error range.
\end{abstract}

Index Terms - non-ridid image registration, mitral annulus tracking, 3D ultrasound

\section{INTRODUCTION}

The mitral annulus is a dynamic, saddle-shaped structure that separates the left atrium from the left ventricle. Mitral annular dynamics play an important role in mitral leaflet coaptation, in reducing mitral leaflet stresses, and in promoting left atrial and left ventricular emptying and filling [1][2]. Several conditions (cardiomyopathy, primary mitral valve pathology, atrial fibrillation) are associated with disturbed annular dynamics, resulting in unfavorable valve competence, leaflet stresses and impaired cardiac function [3].

Analysis of mitral annular dynamics not only provides clinically relevant information in diagnosis and selection of optimal mitral valve repair strategy [4], but is of particular importance in the design and development of new implantable prostheses as well. Furthermore, numerical mitral valve models are emerging as tools to simulate mitral valve closure. Patient-specific annular dynamics are to be imposed as boundary conditions in such numerical models to achieve reliable results [5][6].

Manual tracking of the mitral annulus in volumetric ultrasound images is time consuming and prone to inaccuracy, stressing the need for more automated tracking tools. (Semi-)automatic tracking of annular motion in volumetric ultrasound images has been described earlier using the

\footnotetext{
${ }^{1}$ Henri De Veene and Matthieu De Beule are with IBiTech-bioMMeda, University of Ghent (UGent), 9000 Gent, Belgium and FEops, 9052 Gent, Belgium

${ }^{2}$ Philippe B. Bertrand and Pieter M. Vandervoort are with the Department of Cardiology, Ziekenhuis Oost-Limburg, Genk, Belgium.

${ }^{3}$ Piet Claus, Natasa Popovic and Brecht Heyde are with the Laboratory on Cardiovascular Imaging and Dynamics, University of Leuven (KU Leuven), 3000 Leuven, Belgium, email: brecht.heyde@med.kuleuven.be, piet.claus@uzleuven.be
}

Lucas-Kanade optical flow algorithm [7], with reasonable accuracy [8][9]. The purpose of this study was to evaluate an alternative strategy of automatic mitral annular tracking in real-time 3D ultrasound images (RT3DUS) using non-rigid image registration, and assess its performance in the analysis of annular dynamics for both normal and pathological (secondary mitral regurgitation, SMR) valves.

\section{METHODS}

\section{A. Patient population}

We included 5 consecutive patients with structurally and functionally normal mitral valves that were scheduled to undergo a percutaneous foramen ovale closure, and 5 consecutive patients scheduled to undergo a restrictive mitral annuloplasty because of severe secondary mitral regurgitation at a tertiary referral center (Ziekenhuis Oost-Limburg, Genk, Belgium). Exclusion criteria were presence of atrial fibrillation and poor transesophageal echocardiography windows. The study was approved by the local ethics committee and all patients gave written informed consent.

\section{B. Image acquisition}

RT3DUS images of the mitral valve were prospectively acquired using an X7-t probe on a commercially available workstation (iE33, Philips Medical Systems, Andover, MA). ECG-gated full volume datasets (4 beat interpolation) were acquired during breath-hold to avoid stitching artifacts, starting at the QRS complex (just before MV closure) at an average frame rate of $22 \pm 2 \mathrm{~Hz}$. DICOM images were exported in Cartesian DICOM format using QLab software for further processing.

\section{Manual tracking}

A custom non-commercial tracing package for 3D echocardiograms (Speqle3D), developed at the University of Leuven and tested in an earlier study [10], was used to manually delineate the mitral annulus over time.

Upon loading the images in the software, a local coordinate system was defined in the first frame with origin in the center of the annulus, and 3 orthogonal planes comprising the 3-chamber view (left ventricular outflow tract), the bicommissural view and a short axis view were shown to assist in navigation through the volume. Next, starting from the 3-chamber view, 9 orthogonal long axis planes (rotated in steps of $20^{\circ}$ ) were subsequently used to manually indicate two annular points per plane (Fig. 1a). Using this technique, a clinician manually annotated the cross-section of the annulus 


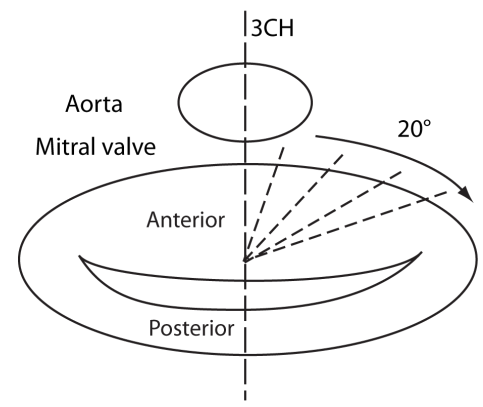

(a) Surgeon's view

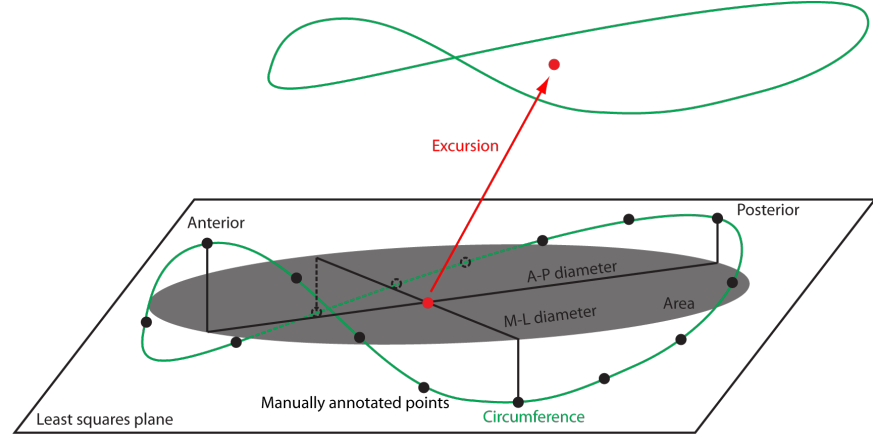

(b) Side view

Fig. 1. Schematic drawing to indicate: (a) the orientation and views to manually contour the mitral valve; (b) the definition of the clinical parameters used to evaluate the tracking algorithm. Abbreviations: A-P = antero-posterior; $\mathrm{M}-\mathrm{L}=$ medio-lateral.

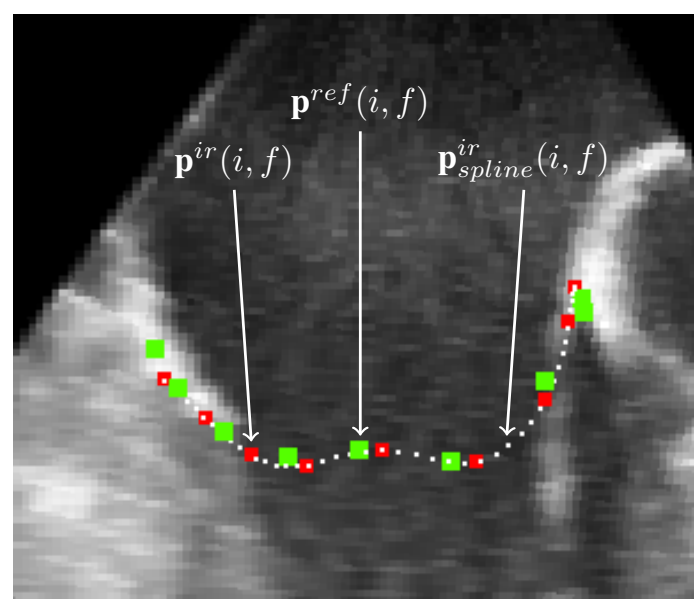

Fig. 2. Example echo dataset where the following is superimposed: (green) manually tracked points $\mathbf{p}^{r e f}(i, f)$, placed in orthogonal long axis cutplanes $\left(20^{\circ}\right.$ steps), (red) automatically tracked points $\mathbf{p}^{i r}(i, f)$, (white) a 3D cubic spline $\mathbf{p}_{\text {spline }}^{i r}(i, f)$ through the automatically tracked points.

$i$ times over the different planes in all frames $f$, leading to a set of reference points $\mathbf{p}^{r e f}(i, f)$ for each patient (Fig. 2).

\section{Automatic tracking}

Non-rigid image registration methods use image warping techniques to estimate motion between images in a sequence. In particular, free-form deformation models where the displacement is modelled using smooth basis functions have been an attractive approach [11]. We have previously described and validated the implementation of this framework within the setting of computing myocardial strain from 3D ultrasound recordings. The reader is referred to [12][13] for more details. In this paper we adopt this strategy for assessing mitral annulus dynamics.

In short, displacement $\mathbf{u}_{F \rightarrow M}(\mathbf{r})$ of a point $\mathbf{r}=[x, y, z]$ between two subsequent images in the sequence, $I_{F}$ (i.e. the fixed image) and $I_{M}$ (i.e. the moving image) was modelled with a three-dimensional third order B-spline tensor-product:

$$
\mathbf{u}_{F \rightarrow M}(\mathbf{r})=\sum_{i \in \mathcal{N}_{i}} \sum_{j \in \mathcal{N}_{j}} \sum_{k \in \mathcal{N}_{k}} \boldsymbol{\mu}^{i j k} \prod_{\xi \in\{x, y, z\}} \beta_{\xi}^{3}\left(\frac{\xi-\kappa_{\xi}^{i j k}}{\sigma_{\xi}}\right)
$$

with $\kappa_{\xi}^{i j k}$ and $\sigma_{\xi}$ the control point location and spacing respectively, and $N_{\xi}$ the set of control points within the compact support $\mathcal{N}$ of the B-spline $\beta_{\xi}(\xi \in\{x, y, z\})$

The optimal displacement field was then found by iteratively optimizing a cost function $C$ consisting of an image similarity metric $S$ and a regularisation term $R$ weighted by the parameter $\omega$. In practice, the sum of squared differences and a smoothness penalty were used respectively:

$$
\begin{gathered}
C\left(I_{F}, I_{M} ; \boldsymbol{\mu}\right)=S\left(I_{F}, I_{M} ; \boldsymbol{\mu}\right)+\omega R(\boldsymbol{\mu}) \\
S=\frac{1}{n} \sum_{\mathbf{r} \in I_{F}}\left[I_{F}(\mathbf{r})-I_{M}(\mathbf{T}(\mathbf{r}))\right]^{2} \\
R=\frac{1}{n} \sum_{\mathbf{r} \in I_{F}}\left\|\frac{\partial^{2} \mathbf{T}(\mathbf{r})}{\partial \mathbf{r} \partial \mathbf{r}^{T}}\right\|_{F}^{2}
\end{gathered}
$$

with $n$ the number of points $\mathbf{r}$ in the image $I_{F}$. Motion in the sequence was estimated in a pairwise fashion. A multiresolution strategy was adopted in which model complexity was gradually increased in 3 steps by halving the grid spacing with every resolution.

The obtained inter-frame displacement fields were then used to propagate the manually annotated points in the first frame $\mathbf{p}^{r e f}(i, 1)$ over the rest of the cardiac cycle, leading to a set of tracked image registration (ir) points $\mathbf{p}^{i r}(i, f)$.

\section{E. Evaluation criteria}

To evaluate the accuracy of the tracking method described above, the root-mean-square error (RMSE) per frame was computed as:

$$
\operatorname{RMSE}(f)=\frac{1}{N} \sqrt{\sum_{i=1}^{N}\left|\mathbf{p}^{r e f}(i, f)-\mathbf{p}_{\text {spline }}^{\text {ir }}(i, f)\right|^{2}}
$$

where $N$ is the number of annotated annulus points per frame. It should be noted that the points $\mathbf{p}^{\text {ref }}(i, f)$ lie in fixed planes. Given that the tracking is performed in 3D, the automatically tracked points $\mathbf{p}^{i r}(i, f)$ follow the anatomical movement and thus move out of these fixed planes. In order to avoid biasing the results in this way, the distance in Eq. (5) was therefore calculated between $\mathbf{p}^{\text {ref }}(i, f)$ and the 
closest point on a cubic spline $\mathbf{p}_{\text {spline }}^{\text {ir }}(i, f)$ drawn through the tracked points $\mathbf{p}^{i r}(i, f)$ (Fig. 2).

The RMSE was used to finetune the smoothness penalty weight $\omega$ and the grid spacing $\sigma$. One dataset was used for training, in which both parameters were changed independently. The optimal parameters were then used as input for the tracking algorithm to process the remainder of the datasets.

The clinical performance of the tracking algorithm was assessed by computing several parameters (Fig. 1b). The circumference was defined as the length of the spline representing the annulus through the tracked points. Next, a least square fitting plane to the annulus was estimated. The area of the annulus was defined by its projection onto this plane. The long and short diameter of the projected area form the antero-postero (AP), and medio-lateral (ML) diameter. Finally, annular excursion was determined as the movement of the annulus centroid over time.

In order to define the clinically acceptable accuracy for tracking, intraobserver variability in delineating the mitral annulus was determined. The same clinician was asked to repeat manual segmentation in the middle of the acquired cycle (just before valve closure) and at the end of the acquired cycle (just before the valve opens), 3 weeks after - and blinded for — the first segmentation.

\section{F. Statistical Analysis}

Results are expressed as mean \pm standard deviation. Continuous variables were compared using the paired and unpaired two-tailed Student t-test when appropriate. Statistical significance was set at a two-tailed probability of $\mathrm{p}<0.05$. Statistical analyses were performed using the Statistical Package for Social Sciences release 20.0 (SPSS Inc., Chicago, Illinois, USA).

\section{RESULTS}

Fig. 3 shows the optimization of the smoothness penalty weight (parameter $\omega$ ) within the interval $1 \cdot 10^{4}$ to $1 \cdot 10^{7}$. An optimal value with respect to RMSE was obtained for a value of $5 \cdot 10^{5}$. The grid spacing parameter at the finest model resolution $\sigma$ was chosen to be isotropic, and was modified within the range of 6 to 14 pixels. With only a maximum change of $2 \%$ in RMSE for the assessed range, this parameter did not have a significant influence on the tracking for this particular dataset. All remaining datasets were tracked using an isotropic gridspacing of 8 pixels at the finest resolution level.

With the optimal parameters, the tracking algorithm resulted in an average RMSE of $1.96 \pm 0.46 \mathrm{~mm}$ over all patients. Table I summarizes the annular dynamics during one cardiac cycle for the patient population. Fig. 4 shows example curves of the relative area change (\%) during one cardiac cycle for a healthy valve versus an SMR patient using both the manual and automatic tracking. Finally, the intraobserver RMSE for the 10 datasets was $1.59 \pm 0.38 \mathrm{~mm}$.

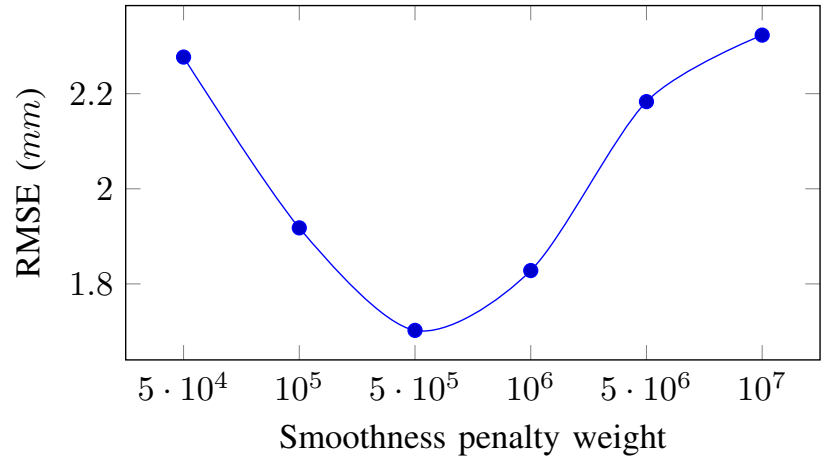

Fig. 3. The RMSE averaged out over all frames of a cardiac cycle of one dataset for different values of the smoothness penalty weight $\omega$.

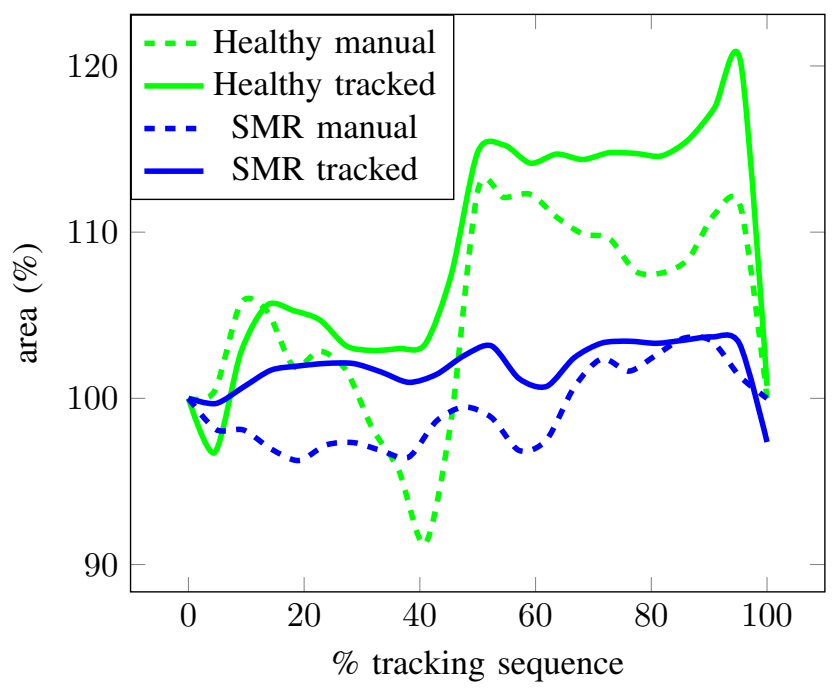

Fig. 4. The relative area change over the course of the cardiac cycle for one normal and one pathological dataset both for the manual tracking and automatic tracking.

\section{DISCUSSION}

This study demonstrated the feasibility of automatically tracking the mitral annulus during the cardiac cycle in volumetric ultrasound images using an algorithm based on nonrigid image registration. Our results show realistic automatic tracking curves, using only a manually delineated mitral annulus in the first frame of the sequence as input. We obtained a RMSE of $1.96 \pm 0.46 \mathrm{~mm}$ over all datasets. Interestingly, the RMSE tended to be lower for the SMR patients compared to healthy valves $(1.76 \pm 0.43 \mathrm{~mm}$ vs $2.16 \pm 0.43, p=0.17)$. Given that these patients tend to show less annular dynamics during the cardiac cycle compared to healthy MV subjects (Fig. 4, Table I), inter-frame motion is typically lower and therefore easier to track.

No significant differences in annular dynamics were found as assessed by the automatic tracking compared to the manual delineation (Table I), highlighting the feasibility of our approach. The dynamics also showed that the SMR patient group had a lower area, lower circumference and lower diameter change over the cardiac cycle compared to normals. None of these trends however were significant, most 
TABLE I

ANNULAR DYNAMICS ASSESSED BY THE AUTOMATIC TRACKING VERSUS THE MANUAL TRACKING

\begin{tabular}{rrrrrrr}
\hline & \multicolumn{2}{c}{ Total population $(\mathrm{n}=10)$} & \multicolumn{2}{c}{ Healthy MV $(\mathrm{n}=5)$} & \multicolumn{2}{c}{ SMR $(\mathrm{n}=5)$} \\
\hline Tracking accuracy & \multicolumn{1}{c}{ manual } & algorithm & manual & algorithm & manual & algorithm \\
\hline RMSE over all datasets (mm) & & $1.96 \pm 0.46$ & & $2.16 \pm 0.43$ & & $1.76 \pm 0.43$ \\
\hline Clinical parameters & & & & & & \\
\hline \% Area Change & $16.2 \pm 6.5$ & $14.9 \pm 6.2$ & $19.4 \pm 5.5$ & $17.4 \pm 5.6$ & $12.9 \pm 6.1$ & $12.3 \pm 6.3$ \\
\% Circumference Change & $7.4 \pm 2.3$ & $6.9 \pm 2.8$ & $8.2 \pm 2.3$ & $7.5 \pm 2.1$ & $6.5 \pm 2.1$ & $6.2 \pm 3.5$ \\
\% A-L diameter Change & $15.2 \pm 5.6$ & $14.9 \pm 5.6$ & $16.2 \pm 4.0$ & $18.3 \pm 5.4$ & $14.2 \pm 7.2$ & $11.6 \pm 3.8$ \\
\% M-L diameter change & $9.1 \pm 4.1$ & $7.3 \pm 3.4$ & $11.2 \pm 4.2$ & $9.2 \pm 2.7$ & $6.9 \pm 2.8$ & $5.5 \pm 3.1$ \\
Peak apical excursion (mm) & $6.3 \pm 1.7$ & $5.9 \pm 1.7$ & $7.7 \pm 1.1$ & $7.0 \pm 2.1$ & $4.9 \pm 0.7 \S$ & $4.8 \pm 1.1$ \\
\hline
\end{tabular}

* indicates p-value $<0.05$ for the comparison versus manual tracking

$\S$ indicates p-value $<0.05$ for the comparison versus healthy MV

likely due to the small patient population. The peak apical excursion was significantly lower for the SMR patient group, which is in line with clinical expectations.

The intraobserver RMSE was $1.59 \pm 0.38 \mathrm{~mm}$. This is in line with the reported interobserver variability of a single frame annular delineation in another study [14], where the RMSE was on average $1.65 \pm 0.74 \mathrm{~mm}$ with a range of $0.84-4.16 \mathrm{~mm}$ over 10 observers. As such, the obtained tracking accuracy can be considered to be within clinically acceptable standards.

Several improvements to our existing framework could still be envisioned in order to improve tracking accuracy. First, imposing a torus-shaped mask around the mitral valve would not only speed up tracking, but could also improve tracking since the motion would not negatively be influenced by other tissues surrounding the valve. Secondly, in order to reduce the accumulation of errors over the cardiac cycle, tracking could be performed both forwards and backwards in time, after which both solutions could be combined using a weighing factor.

\section{CONCLUSIONS}

The proposed non-rigid image registration technique allows tracking the mitral annulus with a RMSE of $1.96 \pm$ $0.46 \mathrm{~mm}$, for the total population, and $1.76 \pm 0.43 \mathrm{~mm}$ for the SMR valves, which is within acceptable accuracy in clinical practice. The method was able to automatically provide clinically relevant information, which could be useful in the diagnosis and selection of optimal mitral valve repair strategies.

\section{ACKNOWLEDGMENTS}

B. Heyde and P. Bertrand are post-doctoral and doctoral research fellows of the Research Foundation-Flanders (Belgium, FWO-Vlaanderen) under grant $12 \mathrm{O} 7515 \mathrm{~N}$ and $11 \mathrm{~N} 7214 \mathrm{~N}$ respectively.

\section{REFERENCES}

[1] I. S. Salgo, J. H. Gorman, R. C. Gorman, B. M. Jackson, F. W. Bowen, T. Plappert, M. G. S. J. Sutton, and L. H. Edmunds, "Effect of annular shape on leaflet curvature in reducing mitral leaflet stress," Circulation, vol. 106, no. 6, pp. 711-717, 2002.

[2] J. J. Silbiger, "Anatomy, mechanics, and pathophysiology of the mitral annulus," American heart journal, vol. 164, no. 2, pp. 163-176, 2012.
[3] F. A. Flachskampf, S. Chandra, A. Gaddipatti, R. A. Levine, A. E. Weyman, W. Ameling, P. Hanrath, and J. D. Thomas, "Analysis of shape and motion of the mitral annulus in subjects with and without cardiomyopathy by echocardiographic 3-dimensional reconstruction," Journal of the American Society of Echocardiography, vol. 13, no. 4, pp. 277-287, 2000.

[4] M. M. Levack, A. S. Jassar, E. K. Shang, M. Vergnat, Y. J. Woo, M. A. Acker, B. M. Jackson, J. H. Gorman, and R. C. Gorman, "Threedimensional echocardiographic analysis of mitral annular dynamics implication for annuloplasty selection," Circulation, vol. 126, no. 11 suppl 1, pp. S183-S188, 2012.

[5] M. Stevanella, E. Votta, and A. Redaelli, "Mitral valve finite element modeling: implications of tissues nonlinear response and annular motion," Journal of biomechanical engineering, vol. 131, no. 12, p. 121010, 2009

[6] Y. Rim, D. D. McPherson, K. B. Chandran, and H. Kim, "The effect of patient-specific annular motion on dynamic simulation of mitral valve function," Journal of biomechanics, vol. 46, no. 6, pp. 1104-1112, 2013.

[7] B. D. Lucas and T. Kanade, "An iterative image registration technique with an application to stereo vision.," in IJCAI, vol. 81, pp. 674-679, 1981.

[8] F. Veronesi, C. Corsi, E. Caiani, L. Sugeng, L. Weinert, V. Mor-Avi, R. Lang, and C. Lamberti, "Semi-automatic tracking for mitral annulus dynamic analysis using real-time 3D echocardiography," in Computers in Cardiology, 2006, pp. 113-116, IEEE, 2006.

[9] R. J. Schneider, N. A. Tenenholtz, D. P. Perrin, G. R. Marx, J. Pedro, and R. D. Howe, "Patient-specific mitral leaflet segmentation from 4D ultrasound," in Medical Image Computing and Computer-Assisted Intervention-MICCAI 2011, pp. 520-527, Springer, 2011.

[10] B. Heyde, D. Barbosa, P. Claus, F. Maes, and J. D'hooge, “Threedimensional cardiac motion estimation based on non-rigid image registration using a novel transformation model adapted to the heart," in STACOM - Statistical Atlases and Computational Models of the Heart, vol. 7746 of LNCS, pp. 142-150, 2012.

[11] D. Rueckert, L. Sonoda, D. Hill, M. Leach, and D. Hawkes, "Nonrigid registration using free-form deformations: application to breast MR images," IEEE Trans Med Imaging, vol. 18, no. 8, pp. 712-721, 1999.

[12] B. Heyde, S. Cygan, H. Choi, B. Lesniak-Plewinska, D. Barbosa, A. Elen, P. Claus, D. Loeckx, K. Kaluzynski, and J. D'hooge, "Regional cardiac motion and strain estimation in three-dimensional echocardiography: A validation study in thick-walled univentricular phantoms," IEEE Trans Ultrason Ferroelectr Freq Control, vol. 59, no. 4, pp. 668-682, 2012.

[13] B. Heyde, S. Bouchez, S. Thieren, M. Vandenheuvel, R. Jasaityte, D. Barbosa, P. Claus, F. Maes, P. Wouters, and J. D'hooge, "Elastic image registration to quantify $3 \mathrm{D}$ regional myocardial deformation from volumetric ultrasound: Experimental validation in an animal model," Ultrasound Med Biol, vol. 39, no. 9, pp. 1688-1697, 2013.

[14] R. J. Schneider, D. P. Perrin, N. V. Vasilyev, G. R. Marx, P. J. del Nido, and R. D. Howe, "Mitral annulus segmentation from 3d ultrasound using graph cuts," IEEE Trans Med Imaging, vol. 29, no. 9, pp. 16761687,2010 